\title{
RETRACTED ARTICLE: Discovery of a novel inhibitory mechanism of kaempferol on TGF- $\beta$ ligand-receptor interaction by single-molecule techniques
}

\author{
Yong Yang $\cdot$ Chunguang Ding $\cdot$ Xiaoyang Li
}

Received: 10 June 2015/ Accepted: 10 September 2015/Published online: 15 September 2015

(C) Springer Science+Business Media Dordrecht 2016

The corresponding author, Yong Yang from China Medical University, would like to retract the article "Discovery of a novel inhibitory mechanism of kaempferol on TGF-? ligand-receptor interaction by single-molecule techniques" since submission has not been authorized by the research supervisor of the author. Moreover, parts of the introduction have been duplicated from the paper "A single-molecule study of the inhibition effect of Naringenin on transforming growth factor-b ligand-receptor binding", 2011, DOI:10.1039/c1cc10778j.

The online version of this article contains the full text of the retracted article as electronic supplementary material.

Electronic supplementary material The online version of this article (doi:10.1007/s10529-015-1959-1) contains supplementary material, which is available to authorized users.

Y. Yang $(\bowtie)$

Institute of Metabolic Disease Research and Drug

Development, China Medical University,

Shenyang 110022, People's Republic of China

e-mail: lovegate5@yahoo.com

C. Ding

Department of Medicinal Chemistry, School of

Pharmaceutical Sciences, Peking University,

Beijing 100191, People's Republic of China

X. Li

Department of Clinical Medicine, Shenyang Medical

College, Shenyang 110034, People's Republic of China
Supporting information Supplementary Fig. 1 Fluorescence intensity by flow cytometry using biotinylated TGF- $\beta 1$ and avidin-FITC.

Supplementary Fig. 2 Single-molecule fluorescence imaging of T $\beta$ RII-GFP monomers and dimers. Distribution of the fluorescence intensities of single GFP molecules imaged on coverslips.

Supplementary Fig. 3 Two representative time courses of T $\beta$ RII-GFP emission show one-step bleaching for monomers and two-step bleaching for dimers.

Supplementary Video 1 Imaging of T $\beta$ RII-GFP molecules on the cell membrane of live HeLa cells. 